\title{
Symmetric Fibonacci Function Solutions of some Nonlinear Partial Differential Equations
}

\author{
YUSUF PANDIR* \\ Bozok University, Department of Mathematics, 66100 Yozgat, Turkey
}

Received: 21 Aug. 2013, Revised: 18 Nov. 2013, Accepted: 19 Nov. 2013

Published online: 1 Sep. 2014

\begin{abstract}
A new version of the Kudryashov's method for solving non-integrable problems in mathematical physics is presented in this paper. New exact solutions of the heat conduction equation and $K(m, n)$ equation with generalized evolution are obtained by using this method. The solutions gained from the proposed method have been verified with obtained by the $\left(G^{\prime} / G\right)$-expansion method.
\end{abstract}

Keywords: heat conduction equation, $K(m, n)$ equation, Kudryashov's method, symmetric Fibonacci functions, exact solutions

\section{Introduction}

The study of nonlinear physical problems has been become very important in recent years. These problems have been solved by using different mathematical approaches. So, soliton solutions, compactons, singular solitons and the other solutions have been found for these physical problems. These types of solutions appear in various areas of physics and engineering. A powerful method for obtaining exact solutions to nonlinear differential equations was proposed in. On the other hand, Kudryashov defined a new method, called as Kudryashov method, in [2]. High order nonlinear evolution equations can be more effectively constructed by using this method. In this research, we give the modified Kudryashov's method combined with symmetrical hyperbolic Fibonacci and Lucas functions for seeking new exact solutions to the non-integrable physical models. We demonstrate applications of the proposed method for two generalized physical problems. One of these equations is the generalized form of heat conduction equation $[3,4]$

$$
u_{t}-b\left(u^{n}\right)_{x x}-b\left(u^{n}\right)_{y y}-u+u^{n}=0,
$$

where $b>0, n>1$, and another equation is the generalized $K(m, n)$ equation $[5,6]$

$$
\left(u^{n}\right)_{t}+\gamma u^{m} u_{x}+\beta\left(u^{n}\right)_{x x x}=0,
$$

In literature, there are also a lot of methods that are used to solve the nonlinear partial differential equations such as the ansatz method [7-11], the exp-function method $[12,13]$, the trial equation method $[14,15]$, the $\left(G^{\prime} / G\right)$-expansion method [16], the Hirota's method $[17,18]$, the three wave method [19], extended Jacobi elliptic function expansion method [20], Kudyrashov method [21], semi-inverse variational principle [22], the multiplier method using the Lie symmetry [23] and many more. Key idea of this paper is that traditional base $e$ of the exponential function is replaced by an arbitrary base $a \neq 1$. So, new exact solutions of non-integrable evolution equations may be obtained by this simple modification.

\section{The modified Kudryashov method}

Suppose that a nonlinear PDE for a function $u$ say in three independent variables $x, y$ and $t$ is given by

$$
P\left(t, x, y, u, u_{t}, u_{x}, u_{y}, u_{t t}, u_{t x}, u_{x x}, u_{y y}, \ldots\right)=0 .
$$

It is useful to summarize the main steps of modified Kudryashov method as follows:

Step 1. Seeking the travelling wave solution of Eq. (3) by the using of the transformations

$$
u(x, y, t)=U(\xi), \quad \xi=k(x+y-c t),
$$

where $k$ and $c$ are free constants. Eq. (3) reduce to the following nonlinear ordinary differential equation:

$$
N\left(U, U^{\prime}, U^{\prime \prime}, \ldots\right)=0,
$$

\footnotetext{
*Corresponding author e-mail: yusufpandir@gmail.com, yusuf.pandir@bozok.edu.tr
} 
where the prime denotes differentiation with respect to $\xi$. Also, it is assumed that the highest order nonlinear terms in Eq. (5) are $U^{l}(\xi) U^{(s)}(\xi)$ and $\left(U^{(p)}\right)^{r}$.

Step 2. We assume that the exact solutions of Eq. (5) have the following form:

$$
U(\xi)=\sum_{j=-N}^{N} a_{j} Q^{j}
$$

where $Q=\frac{1}{1 \pm a^{\xi}}$. We know that the function $Q$ is solution of equation

$$
Q_{\xi}=\ln a\left(Q^{2}-Q\right) .
$$

Step 3. According to the proposed method, Eq. (6) can be expanded as follows:

$U(\xi)=a_{-N} Q^{-N}+\cdots+a_{-1} Q^{-1}+a_{0}+a_{1} Q+\cdots+a_{N} Q^{N}$.

We proceed analogously as in the classical Kudryashov method on balancing the highest order nonlinear terms in Eq. (5) in order to determine the value of $N$. More precisely, by straightforward calculations we get

$$
\begin{gathered}
U^{\prime}(\xi)=\cdots+a_{N} N \ln a Q^{N+1}, \\
U^{\prime \prime}(\xi)=\cdots+a_{N} N(N+1)(\ln a)^{2} Q^{N+2}, \\
U^{(s)}(\xi)=\cdots+a_{N} N(N+1) \ldots(N+s-1)(\ln a)^{s} Q^{N+s}, \\
U^{l} U^{(s)}(\xi)=\cdots+\overline{a_{N}} N(N+1) \ldots(N+s-1)(\ln a)^{s} Q^{(l+1) N+s}, \\
\left(U^{(p)}\right)^{r}(\xi)=\cdots+\left(a_{N} N(N+1) \ldots(N+p-1)(\ln a)^{p}\right)^{r} Q^{r(N+p),}
\end{gathered}
$$

where $a_{N}$ and $\overline{a_{N}}$ are the unknown coefficients. Balancing the highest order nonlinear terms of Eq. (12) and Eq. (13), we obtain

$$
(l+1) N+s=r(N+p)
$$

so

$$
N=\frac{s-r p}{r-l-1}
$$

Step 4. Substituting Eq. (6) into Eq. (5) yields a polynomial $R(Q)$ of $Q$. Equating the coefficients of $R(Q)$ to zero, we get a system of algebraic equations. Solving this system, we shall determine $c, k$ and the arbitary coefficients of $a_{-N}, \ldots, a_{-1}, a_{0}, a_{1}, \ldots, a_{N}$. Thus, we can find the new exact solutions to Eq. (3).

\section{Applications}

Example 1. Application to the generalized form of heat conduction equation

In this section the modified Kudryashov method will be applied to solve heat conduction equation. Using the transformation (4), we can reduce Eq. (1) to the ordinary differential equation which can be written as

$-k c U^{\prime}-b k^{2} n(n-1) U^{n-2} U^{\prime 2}-b k^{2} U^{n-1} U^{\prime \prime}-U+U^{n}=0$.

Then we use the transformation

$$
U(\xi)=V^{\frac{-1}{n-1}}(\xi)
$$

which converts $\mathrm{Eq}$ (16) into

$k c(n-1) V^{\prime} V^{2}+2 b k^{2} n(1-2 n) V^{\prime 2}+2 b k^{2} n(n-1) V V^{\prime \prime}-(n-1)^{2} V^{3}+(n-1)^{2} V^{2}=0$.

We take

$$
V(\xi)=\sum_{j=-N}^{N} a_{j} Q^{j},
$$

where $Q=\frac{1}{1 \pm a^{\xi}}$ and $Q_{\xi}=\ln a\left(Q^{2}-Q\right)$. Using the generalized balance formula (15) for the nonlinear terms $V V^{\prime \prime}$ and $V^{\prime} V^{2}$ in Eq. (18), we find

$$
N=1 \text {. }
$$

Therefore we have

$$
V(\xi)=\sum_{j=-1}^{1} a_{j} Q^{j}=\frac{a_{-1}}{Q}+a_{0}+a_{1} Q,
$$

and we compute derivatives of the function $y(\xi)$ with respect to $\xi$. The required derivatives in Eq (18) are obtained

$$
\begin{gathered}
V_{\xi}=\ln a\left(\frac{a_{-1}}{Q}-a_{-1}+a_{1} Q+a_{1} Q^{2}\right), \\
V_{\xi \xi}=\ln ^{2} a\left(\frac{a_{-1}}{Q}-a_{-1}+a_{1} Q-3 a_{1} Q^{2}+2 a_{1} Q^{3}\right) .
\end{gathered}
$$

As result of this, we get the system of algebraic equations can be solved by using Mathematica. Solving this system, we obtain coefficients $a_{-1}, a_{0}$ and $a_{1}$ as follows:

\section{Case 1}

$a_{0}=-a_{-1}, \quad a_{1}=0, \quad c= \pm n \sqrt{2 b}, \quad k=\mp \frac{1-n}{n \sqrt{2 b} \ln a}$,

where $a_{-1}$ is arbitrary constant. By the using Eq. (24), Eq. (21) can be written as

$$
V(\xi)=a_{-1} a^{\xi},
$$

where $\xi=\mp \frac{1-n}{n \sqrt{2 b} \ln a}(x+y \pm n \sqrt{2 b} t)$. Therefore, we obtain the following solution of $\mathrm{Eq}$ (1)

$$
u_{1}(x, y, t)=A\left( \pm a^{ \pm \frac{1}{n \sqrt{2 b} \ln a}(x+y \pm n \sqrt{2 b} t)}\right)^{\frac{1}{1-n}}
$$

where $A=\left(a_{-1}\right)^{\frac{1}{1-n}}$. 


\section{Case 2}

$a_{-1}=a_{0}=0, \quad a_{1}=1, \quad c= \pm \sqrt{2 b}, \quad k=\mp \frac{(1-n)}{n \sqrt{2 b} \ln a}$.

Using Eq. (27), we obtain the following solution for $\xi=$ $\mp \frac{1-n}{n \sqrt{2 b} \ln a}(x+y \pm \sqrt{2 b} t)$

$$
V(\xi)=\frac{1}{1 \pm a^{\xi}} .
$$

Substituting Eq. (28) into (17) and (4), respectively, we have

$$
\begin{aligned}
& u_{2}(x, y, t)=\left(\frac{1}{1+a^{\mp \frac{1-n}{n \sqrt{2 b} \ln a}}(x+y \pm \sqrt{2 b} t)}\right)^{\frac{1}{1-n}}, \\
& u_{3}(x, y, t)=\left(\frac{1}{1-a^{\mp \frac{1-n}{n \sqrt{2 b} \ln a}(x+y \pm \sqrt{2 b} t)}}\right)^{\frac{1}{1-n}} .
\end{aligned}
$$

Applying several simple transformations to these solutions, we obtain new exact solutions to Eq. (1), respectively:

$$
\begin{aligned}
& u_{2}(x, y, t)=\left(\frac{1}{2}-\frac{1}{2} \mathrm{tFs}[B(x+y-c t)]\right)^{\frac{1}{1-n}}, \\
& u_{3}(x, y, t)=\left(\frac{1}{2}-\frac{1}{2} \operatorname{ctFs}[B(x+y-c t)]\right)^{\frac{1}{1-n}},
\end{aligned}
$$

where $B=\mp \frac{1-n}{2 n \sqrt{2 b} \ln a}, c= \pm \sqrt{2 b}$.

Case 3

$a_{-1}=0, \quad a_{0}=1, \quad a_{1}=-1, \quad c= \pm \sqrt{2 b}, \quad k=\mp \frac{1-n}{n \sqrt{2 b} \ln a}$.

Using Eq. (33), we obtain the following solution for $\xi=$ $\mp \frac{1-n}{n \sqrt{2 b} \ln a}(x+y \pm \sqrt{2 b} t)$

$$
V(\xi)=\frac{a^{\xi}}{1+a^{\xi}} .
$$

Then we get the exact solutions of generalized heat conduction equation as

$$
u_{4}(x, y, t)=\left(\frac{a^{\mp \frac{1-n}{n \sqrt{2 b} \ln a}(x+y \pm \sqrt{2 b} t)}}{1+a^{\mp \frac{1-n}{n \sqrt{2 b} \ln a}(x+y \pm \sqrt{2 b} t)}}\right)^{\frac{1}{1-n}} .
$$

Remark 1. The solutions (3.11), (3.16), (3.17) and (3.20) are new and more general solutions for the generalized forms of the nonlinear heat conduction equation. These solutions have been obtained by the $\left(G^{\prime} / G\right)$-expansion method in [3] and [4], but our results are more general than it.

Example 2. Application to the generalized form of $K(m, n)$ equation
Using the transformations

$$
u(x, t)=U(\xi), \quad \xi=k(x-c t)
$$

and substituting Eq. (36) into Eq. (2), we can obtain

$$
-k c\left(U^{n}\right)_{\xi}+\gamma k U^{m} U_{\xi}+\beta k^{3}\left(U^{n}\right) \xi \xi \xi=0 .
$$

Integrating Eq. (37) with respect to $\xi$ once yields

$$
-k c\left(U^{n}\right)+\frac{\gamma k U^{m+1}}{m+1}+\beta k^{3}\left(U^{n}\right) \xi \xi+C=0,
$$

where $C$ is the integration constant. For simplicity, we take $C=0$ and use the relation

$$
U(\xi)=V^{\frac{1}{m-n+1}}(\xi),
$$

which will convert Eq (38) into

$$
\begin{gathered}
\beta k^{3} n(m-n+1)(m+1) V V^{\prime \prime}+\beta k^{3} n(2 n-m-1)(m+1)\left(V^{\prime}\right)^{2} \\
-k c(m+1)(m-n+1)^{2} V^{2}+\gamma k(m-n+1)^{2} V^{3}=0 .
\end{gathered}
$$

According to the solution procsedure, we can compute the value $N$ from the balance formula (15) for the nonlinear terms $V V^{\prime \prime}$ and $V^{3}$ in Eq (40) as follows:

$$
N=2 \text {. }
$$

Therefore we have

$$
V(\xi)=\sum_{j=-2}^{2} a_{j} Q^{j}=\frac{a_{-2}}{Q^{2}}+\frac{a_{-1}}{Q}+a_{0}+a_{1} Q+a_{1} Q^{2},
$$

and we substitute derivatives of the function $y(\xi)$ with respect to $\xi$. The required derivatives in $\mathrm{Eq}(40)$ are obtained

$$
\begin{gathered}
V_{\xi}=\ln a\left(\frac{a_{-1}}{Q}-a_{-1}+a_{1} Q+a_{1} Q^{2}\right), \\
V_{\xi \xi}=\ln ^{2} a\left(\frac{a_{-1}}{Q}-a_{-1}+a_{1} Q-3 a_{1} Q^{2}+2 a_{1} Q^{3}\right) .
\end{gathered}
$$

As result of this, we have the system of algebraic equations can be solved with Mathematica. Solving this system, we obtain the following results:

$$
\begin{aligned}
& a_{-2}=a_{-1}=a_{0}=0, \quad a_{1}=\frac{2 \beta n k^{2}(\ln a)^{2}(1+m)(m+n+1)}{\gamma(m-n+1)^{2}}, \\
& a_{2}=\frac{-2 \beta n k^{2}(\ln a)^{2}(1+m)(m+n+1)}{\gamma(m-n+1)^{2}}, \quad c=\frac{\beta k^{2} n^{2}(\ln a)^{2}}{(-1-m+n)^{2}},
\end{aligned}
$$

where $a_{1}, a_{2}$ are arbitrary constants. By the using Eq. (45), Eq. (42) can be writen as

$$
V(\xi)=\frac{2 \beta n k^{2}(\ln a)^{2}(1+m)(m+n+1)}{\gamma(m-n+1)^{2}}\left(\frac{1}{1 \pm a^{\xi}}-\frac{1}{\left(1 \pm a^{\xi}\right)^{2}}\right),
$$



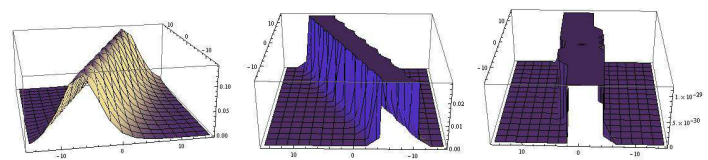

Fig. 1: $u_{1}(x, t)$ is shown at $a=b=n=k=m=1$.
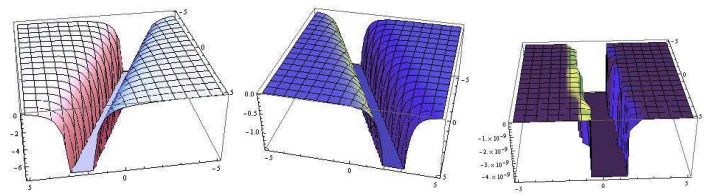

Fig. 2: $u_{2}(x, t)$ is shown at $a=b=n=k=m=1$.

where $\xi=k\left(x-\frac{\beta k^{2} n^{2}(\ln a)^{2}}{(-1-m+n)^{2}} t\right)$. Substituting Eq. (46) into Eq. (39), we have

$U(x, t)=A\left(\frac{1}{1 \pm a^{k\left(x-\frac{\beta k^{2} n^{2}(\ln a)^{2}}{(-1-m+n)^{2}} t\right)}}-\frac{1}{\left(1 \pm a^{\left.k\left(x-\frac{\beta k^{2} n^{2}(\ln a)^{2}}{(-1-m+n)^{2}} t\right)\right)^{2}}\right.}\right)^{\frac{1}{m-n+1}}$

where $A=\left(\frac{2 \beta n k^{2}(\ln a)^{2}(1+m)(m+n+1)}{\gamma(m-n+1)^{2}}\right)^{\frac{1}{m-n+1}}$. Applying several simple transformations to these solutions, we obtain new exact solutions to Eq. (2), respectively:

$$
\begin{aligned}
& u_{1}(x, t)=\frac{\tilde{A}}{\mathrm{cFs}^{\frac{2}{m-n+1}}[B(x-c t)]}, \\
& u_{2}(x, t)=\frac{-\tilde{A}}{\operatorname{sFs}^{\frac{2}{m-n+1}}[B(x-c t)]},
\end{aligned}
$$

where $\tilde{A}=\left(\frac{2 \beta n k^{2}(\ln a)^{2}(1+m)(m+n+1)}{5 \gamma(m-n+1)^{2}}\right)^{\frac{1}{m-n+1}}, B=\frac{k}{2}$ and $c=\frac{\beta k^{2} n^{2}(\ln a)^{2}}{(-1-m+n)^{2}}$. Here, $A$ represents the amplitude of the solitons, while $\mathrm{B}$ is the inverse width of the solitons and $c$ represents the velocity of the solitons. Also, Eq. (49) represents a singular soliton solution for Eq. (2). If we take $a=e$ in Eq. (48), then we can find the solution obtained by the ansatz method in [5].

\section{Remarks and Conclusion}

In this section is to show that the symmetrical hyperbolic Fibonacci function and Lucas function solutions can be obtained by the general Expa-function with Kudryashov method. We highlight briefly the definitions of symmetrical hyperbolic Fibonacci and Lucas functions. Stakhov and Rozin gave all details of symmetrical hyperbolic Fibonacci and Lucas functions in [24]. We only examine several formulas with respect to these functions here. Symmetrical Fibonacci sin, cosine, tangent and cotangent functions are respectively defined as follows:

$$
\begin{gathered}
s F s(x)=\frac{a^{x}-a^{-x}}{\sqrt{5}}, \quad c F s(x)=\frac{a^{x}+a^{-x}}{\sqrt{5}}, \\
t F s(x)=\frac{a^{x}-a^{-x}}{a^{x}+a^{-x}}, \quad c t F s(x)=\frac{a^{x}+a^{-x}}{a^{x}-a^{-x}} .
\end{gathered}
$$

Analogously, symmetrical Lucas sin and cosine are respectively defined as

$$
s L s(x)=a^{x}-a^{-x}, \quad c L s(x)=a^{x}+a^{-x},
$$

where $a=\frac{1+\sqrt{5}}{2}$, which is known in the literature as Golden Mean [1]. So, we can find more general (or more larger classes of) solutions in applying the modified method with Symmetrical Fibonacci functions. If we take $a=e$, then we can find the previous solutions also.

\section{Acknowledgement}

The research has been supported by Yozgat University Foundation.

\section{References}

[1] T. A. Ahmad and R. H. Ezzat, Applied Mathematics and Computation, 217, 451-459 (2010).

[2] N. A. Kudryashov, Communications in Nonlinear Science and Numerical Simulation, 17, 2248-2253 (2012).

[3] M. M. Kabir, Nonlinear Analysis: Real World Applications, 12, 2681-2691 (2011).

[4] M. M. Kabir, Exact Travelling Wave Solutions for Generalized Forms of the Nonlinear Heat Conduction Equation, Heat Conduction-Basic Research, Edited by Vyacheslav S. Vikhrenkod, InTech, (2011).

[5] A. Biswas, Physics Letters A, 372, 4601-4602 (2008).

[6] G. Ebadi and A. Biswas, Communications in Nonlinear Science and Numerical Simulation, 16, 2377-2382 (2011).

[7] A. Biswas, International Journal of Theoretical Physics, 48, 2698-2703 (2009).

[8] A. Biswas, Computers and Mathematics with Applications, 59, 2536-2540 (2010).

[9] Anjan Biswas, Chenwi Zony, Essaid Zerrad, Soliton perturbation theory for the quadratic nonlinear KleinGordon equation, Applied Mathematics and Computation, 203, 153156 (2008).

[10] Luca Maragliano, Alexander Fischer, Eric Vanden-Eijnden and Giovanni Ciccotti, String method in collective variables: Minimum free energy paths and isocommittor surfaces, The Journal of Chemical Physics, 125, (2006).

[11] V. N. Serkin, V.M. Chapela, J. Percino, T.L. Belyaeva, Nonlinear tunneling of temporal and spatial optical solitons through organic thin films and polymeric waveguides, Optics Communications, 192, 237-244 (2001). 
[12] Y. Gurefe and E. Misirli, Computers and Mathematics with Applications, 61, 2025-2030 (2011).

[13] E. Misirli and Y. Gurefe, Mathematical and Computational Applications, 16, 258-266 (2011).

[14] Y. Gurefe, A. Sonmezoglu and E. Misirli, Pramana-Journal of Physics, 77, 1023-1029 (2011).

[15] Y. Gurefe, A. Sonmezoglu and E. Misirli, Journal of Advanced Mathematical Studies, 5, 41-47 (2012).

[16] Y. Gurefe and E. Misirli, Applied Mathematics and Computation, 217, 9189-9197 (2011).

[17] A. H. Salas, O. G. Hurtado and J. E. Castillo, International Journal of Physical Sciences, 6, 7729-7737 (2011).

[18] Y. Gurefe, Y. Pandir and E. Misirli, Applied Mathematical Sciences, 6, 3225-3234 (2012).

[19] Z. D. Dai, C. J. Wang, S. Q. Lin, D. L. Li and G. Mu, Nonlinear Science Letters A, 1, 77-82 (2010).

[20] Y. Xiao, H. Xue, H. Zhang, Journal of Applied Mathematics, Article ID 896748, 2012, (2012).

[21] H. Bulut and B. Kili, NWSA-Physical Sciences, 8, 24-31 (2013).

[22] A. Biswas, Quantum Physics Letters, 1, 79-86 (2012).

[23] Nikolai A. Kudryashov, Dmitry I. Sinelshchikov, Maria V. Demina, Exact solutions of the generalized Bretherton equation, Physics Letters A, 375, 1074-1079 (2011).

[24] A. Stakhov and B. Rozin, Chaos, Solitons and Fractals, 23, 379-389 (2005)

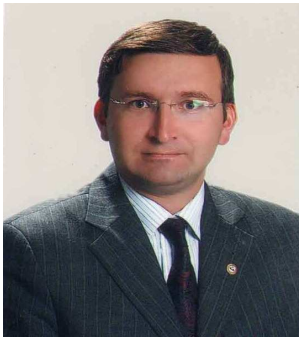

Y. Pandir is an assistant professor in Department of Mathematics at Bozok University; Yozgat (Turkey). $\mathrm{He}$ obtained his M.Sc. degree from Celal Bayar University and Ph.D. degree from Erciyes University. His research interests include fluid mechanics, finite element method, analytical methods for nonlinear differential equations, mathematical physics, and numerical analysis. His research papers are published in Abstract and Applied Analysis, Applied Mathematical Sciences, International Journal of Physical Sciences, Physica Scripta and Journal of Mathematics, Discrete Dynamics in Nature and Society, Life Science Journal. 